\begin{tabular}{|c|c|c|}
\hline INESEG & $\begin{array}{l}\text { INTERNATIONAL } \\
\text { ENGINEERING, } \\
\text { SCIENCE AND } \\
\text { EDUCATION } \\
\text { GROUP }\end{array}$ & $\begin{array}{l}\text { International Journal of Health Services Research and Policy } \\
\text { (2018) 3(3): 117-122 } \\
\text { Published online December, } 2018 \text { (http://dergipark.gov.tr/ijhsrp) } \\
\text { doi: } 10.23884 \text { ijhsrp.2018.3.3.03 } \\
\text { e-ISSN: } 2602-3482 \\
\text { Received: November 10, } 2018 \quad \text { Accepted: December 12, } 2018 \\
\text { Submission Type: Research Article }\end{array}$ \\
\hline
\end{tabular}

\title{
EVALUATION OF DIASTOLIC DYSFUNCTION IN RHEUMATOID ARTHRITIS PATIENTS
}

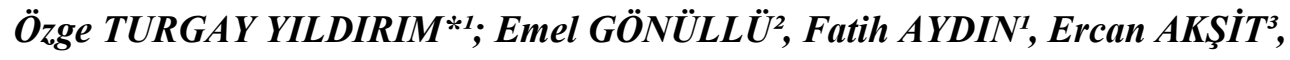 \\ Ayşe HÜSEYINOĞLU AYDIN ${ }^{1}$ Evrin DAĞTEKİN \\ ${ }^{1}$ Eskisehir State Hospital, Cardiology Department, Eskisehir, Turkey \\ ${ }^{2}$ Eskisehir State Hospital, Rheumatology Department, Eskisehir, Turkey \\ ${ }^{3}$ Çanakkale Onsekiz Mart University Faculty of Medicine, Cardiology Department, Çanakkale, Turkey \\ *Corresponding author; ozgeturgay@gmail.com
}

\begin{abstract}
Rheumatoid arthritis (RA) is a chronic, autoimmune inflammatory disorder which can also affect cardiovascular system. Cardiovascular system involvement can be seen in many forms such as pericarditis, myocarditis, coronary artery disease or heart failure. Diastolic dysfunction is especially important for preserved ejection fraction heart failure patients. With this study we aim to investigate echocardiographic diastolic dysfunction parameters in RA patients. 77 RA patients and 76 control subjects without coronary artery disease, heart failure, valvular heart diseases were included for the study and echocardiography was performed. Interventricular septum $(p=0.041)$, posterior wall diameter $(p=0.008)$, left ventricular mass index $(p=0.037)$ values were significantly higher and $E$ wave $(p=0.000), E / A(p=0.000), e$, $(p=0.018)$ values were significantly lower in $R A$ patients compared to control group. In conclusion echocardiographic diastolic dysfunction parameters were declined in RA patients.
\end{abstract}

Key words: Rheumatoid arthritis, Diastolic dysfunction, Echocardiography

\section{Introduction}

Rheumatoid arthritis (RA) is a chronic, systemic, autoimmune inflammatory disorder. Although RA primarily affects the peripheral joints, extra-articular involvement is also seen in this disorder. RA may affect many systems such as cardiovascular, ocular, neurological, hematological and pulmonary systems [1]. Early detection for cardiovascular involvement is very important because cardiovascular diseases are the major cause of mortality in RA patients [2,3]. Cardiovascular involvement can be seen in different forms such as pericarditis, myocarditis, coronary artery disease, heart failure and rhythm disorders [4]. Heart failure incidence is higher in RA patients and $13 \%$ of mortality is caused by heart failure $[5,6]$. The risk of coronary artery disease is two times higher in RA patients compared to normal population [7]. Autoimmunity and inflammation along with traditional risk factors contribute to the development of cardiovascular diseases [8]. This may explain the higher incidence in heart failure. Also amyloidosis or antirheumatic therapy may play roles for the development of heart failure [9]. 
Left ventricular diastolic dysfunction (LVDD) is associated with increased left ventricular filling pressure and left atrial volume [10]. Evaluation of LVDD is especially important for heart failure patients with preserved ejection fraction [11]. Since coronary artery disease and heart failure are more common in RA, detection of disrupted LVDD is especially important for this group of patients. With this study we aim to investigate the LVDD parameters via echocardiography in RA patients.

\section{Methods}

77 patients with diagnosis of RA according to the American College of Rheumatology criteria and 76 age and sex-matched control subjects were included for the cross-sectional study. All RA patients were under treatment and their diseases were not in active state. The patients below the age of 18, with severe valvular heart disease, heart failure or coronary artery disease were excluded. Brief anamnesis was conducted to obtain demographic data, duration of disease, history of chronic diseases. Weight and height data were collected while patients were wearing only their underwear.

The study was approved by the local ethics committee. Informed consent was obtained from each patient.

A 2-dimensional and Doppler echocardiography was performed at the admission to the clinic. Echocardiographic evaluation was performed according to guidelines of American Society of Echocardiography. Ejection fraction (EF), left ventricular end diastolic diameter (LVEDD), interventricular septal (IVS) thickness, posterior wall (PW) thickness, the ratio of peak velocity flow in early diastole to peak velocity flow in the late diastole (E/A), the ratio of $\mathrm{E}$ to early diastolic mitral annular velocity (E/e'), left atrium diameter, left atrium volume were examined. Left ventricular mass was calculated using Devereux formula and left ventricular mass index was calculated by left ventricular mass/body surface area [12].

Blood sample was taken in the morning after 8 hours of fasting to determine glucose, urea, creatinine, HDL-C, LDL-C, triglycerides, hemoglobin levels, leukocyte, neutrophil, C-reactive protein (CRP), PDW values.

Data were presented as mean \pm standard deviation (SD) and as proportions for categorical variables. The t-test or Chi-square test was used for comparisons of continuous and categorical variables, respectively. Distribution of the data for normality was tested by the Shapiro-Wilk test and homogeneity of group variances were tested by the Levene test. For the parameters which are not normally distributed, Mann Whithey U test was used. The data were analyzed using IBM SPSS ver. 22.0.

\section{Results}

A total of 153 patients were enrolled for the study. The study population included 77 patients with RA (50 males; mean age, $53.1 \pm 7.6$ years) and 76 healthy subjects as controls (51 males; mean age, $50.5 \pm 11.8$ years). The groups were similar in regards of age, gender, hypertension, diabetes mellitus, smoking status, weight, height, body-mass index, fasting blood sugar, urea, creatinine, highdensity lipoprotein cholesterol, low-density lipoprotein cholesterol, triglyceride and hemoglobin levels ( $p>0.05)$. There were significant differences in white blood cell $(p=0.003)$, neutrophil counts $(p=0.003)$ and CRP levels. Table 1 shows the patients' clinical data and laboratory results.

Table 1. Clinical and Biochemical Characteristics of Study Groups

\begin{tabular}{|c|c|c|c|}
\hline & $\begin{array}{c}\text { Rheumatoid Arthritis } \\
\text { Patients }(\mathrm{n}=77)\end{array}$ & Control Group $(\mathrm{n}=76)$ & $\mathrm{p}$ \\
\hline
\end{tabular}




\begin{tabular}{|l|c|c|c|}
\hline Age, years & $53.1 \pm 7.6$ & $50.5 \pm 11.8$ & 0.162 \\
\hline Gender, Male/Female & $50 / 27$ & $51 / 25$ & 0.777 \\
\hline Hypertension, \% & $15(19.5 \%)$ & $20(26.6 \%)$ & 0.293 \\
\hline Diabetes Mellitus, \% & $15(19.4 \%)$ & $7(9.2 \%)$ & 0.070 \\
\hline Smoking, \% & $25(32.5 \%)$ & $26(34.2 \%)$ & 0.829 \\
\hline Weight, kg & $74.2 \pm 12.0$ & $75.6 \pm 13.5$ & 0.494 \\
\hline Height, cm & $164.8 \pm 8.2$ & $165.0 \pm 8.7$ & 0.871 \\
\hline BMI, kg/m ${ }^{2}$ & $27.3 \pm 4.2$ & $27.8 \pm 5.1$ & 0.504 \\
\hline Blood Examination & & & \\
\hline FBG, mg/dl & $101.5 \pm 31.3$ & $96.6 \pm 23.8$ & 0.283 \\
\hline Urea, mg/dl & $13.1 \pm 3.7$ & $12.8 \pm 4.2$ & 0.707 \\
\hline Creatinine, mg/dl & $0.7 \pm 0.1$ & $0.7 \pm 0.1$ & 0.328 \\
\hline HDL-C, mg/dl & $48.9 \pm 8.4$ & $53.5 \pm 12.7$ & 0.166 \\
\hline LDL-C, mg/dl & $116.4 \pm 48.8$ & $123.8 \pm 32.6$ & 0.577 \\
\hline TG, mg/dl & $148.0 \pm 57.6$ & $144.4 \pm 94.1$ & 0.879 \\
\hline Hb, g/dl & $13.6 \pm 1.7$ & $14.1 \pm 1.7$ & 0.078 \\
\hline WBC counts & $9123.6 \pm 3231.3$ & $7782.5 \pm 1973.6$ & 0.003 \\
\hline Neutrophil counts & $5844.6 \pm 2699.7$ & $4732.1 \pm 1709.8$ & 0.003 \\
\hline CRP, mg/dl & $1.1 \pm 1.6$ & $0.6 \pm 0.5$ & 0.013 \\
\hline
\end{tabular}

Abbreviations: BMI, body-mass index; CRP, C-reactive protein; FBS, fasting blood sugar; HDL-C, high-density lipoprotein cholesterol; Hb, hemoglobin; LDL-C, low-density lipoprotein cholesterol; RDW, red cell distribution width; TG, triglyceride; WBC, white blood cell.

Ejection fraction, LVEDD, left atrium diameter, left atrium volume, A wave and E/e' values were statistically similar between RA patients and control group ( $>>0.05)$. Interventricular septum $(\mathrm{p}=0.041)$, $\mathrm{PW}$ diameter $(0.008)$, left ventricular mass index $(\mathrm{p}=0.037)$ values were significantly higher and $E$ wave $(p=0.000), E / A(p=0.000)$, e' $(0.018)$ values were significantly lower in RA patients compared to control group (Table 2).

Table 2. Echocardiography results of the study population

\begin{tabular}{|l|c|c|c|}
\hline Echocardiography Results & $\begin{array}{c}\text { Rheumatoid Arthritis } \\
\text { Patients }(\mathrm{n}=77)\end{array}$ & $\begin{array}{c}\text { Control Group } \\
(\mathrm{n}=76)\end{array}$ & $\mathrm{p}$ \\
\hline Ejection fraction, \% & $58.0 \pm 6.1$ & $57.1 \pm 2.8$ & 0.299 \\
\hline LVEDD, mm & $43.9 \pm 4.8$ & $44.5 \pm 4.3$ & 0.472 \\
\hline IVS diameter, mm & $9.6 \pm 1.8$ & $8.9 \pm 2.3$ & 0.041 \\
\hline PW diameter, mm & $8.9 \pm 1.6$ & $8.1 \pm 1.8$ & 0.008 \\
\hline
\end{tabular}




\begin{tabular}{|c|c|c|c|}
\hline Left atrium diameter, $\mathrm{mm}$ & $33.8 \pm 5.0$ & $32.6 \pm 3.8$ & 0.085 \\
\hline Left atrium volume, $\mathrm{cm}^{3}$ & $28.2 \pm 8.3$ & $27.1 \pm 8.9$ & 0.451 \\
\hline Left ventricular mass index, $\mathrm{g} / \mathrm{m}^{2}$ & $73.1 \pm 18.2$ & $66.5 \pm 20.1$ & 0.037 \\
\hline \multicolumn{4}{|l|}{ Diastolic Dysfunction parameters } \\
\hline $\mathrm{E}, \mathrm{cm} / \mathrm{s}$ & $60.4 \pm 13.3$ & $70.6 \pm 15.0$ & 0.000 \\
\hline $\mathrm{A}, \mathrm{cm} / \mathrm{s}$ & $68.3 \pm 15.2$ & $65.4 \pm 15.7$ & 0.254 \\
\hline $\mathrm{E} / \mathrm{A}$ & $0,9 \pm 0.3$ & $1.1 \pm 0.3$ & 0.000 \\
\hline $\mathrm{e}^{\prime}, \mathrm{cm} / \mathrm{s}$ & $8.3 \pm 2.7$ & $9.5 \pm 2.8$ & 0.018 \\
\hline E/e’ & $7.8 \pm 2.4$ & $8.0 \pm 2.4$ & 0.707 \\
\hline
\end{tabular}

Abbreviations: A, mitral A wave; E, mitral E wave; e', average of lateral e' and septal e'; E/A, ratio of mitral $\mathrm{E}$ wave to mitral A wave; E/e', ratio of mitral $\mathrm{E}$ wave to average of lateral e' and septal e'; IVS, interventricular septum; LVEDD, left ventricular end diastolic diameter; PW, posterior wall.

\section{Discussion}

We evaluated the diastolic dysfunction parameters with this study. We found out that diastolic dysfunction parameters such as E wave, E/A, e' were disrupted; IVS, PW diameters and left ventricular mass index are increased in RA patients compared to normal population.

Rheumatoid arthritis is a common rheumatological disorder worldwide. In RA, cardiovascular involvement can be seen and cardiac manifestations cause increased risk of mortality. Cause of death in $50 \%$ of RA patients is cardiovascular diseases [13].

Long term survival is shorter compared to normal population in RA patients [14]. Prevalence of congestive heart failure is higher and necropsy studies showed pericardial, myocardial and endocardial involvement in RA patients $[15,16]$. All-cause mortality is increased in isolated diastolic dysfunction patients $[17,18]$. In our study we evaluated echocardiographic diastolic dysfunction parameters in RA patients and compared the results with control group. In the study EF results were similar and the patients didn't have known heart failure or cardiovascular diseases. $\mathrm{E}$ wave, E/A ratio and e' values were disrupted and IVS and PW diameters were thicker in RA patients. Di Franco et al. also studied RA patients and found out E/A was disrupted but IVS, PW and other echocardiographic values were similar in RA patients and control group [19]. According to Erdem et al. [20] E wave, E/A, e', IVS and PW results were disrupted in RA patients and these results support our findings. The disruption in transmitral flow suggests myocardial involvement in RA patients [21]. We know that myocardium involvement can be seen as myocarditis or via ischemic heart diseases but in our study ejection fractions of the patients were normal and had no history of coronary artery disease [4]. So the changes in transmitral flow suggest a subclinical involvement of myocardium in RA patients.

Several studies investigating left ventricular mass index in RA patients has been conducted and the results were controversial. Rudominer et al. [22] found out that left ventricular mass index was higher in RA while Myasoedova et al. [23] found the opposite. In Myasodeova et al. study, it was emphasized that left ventricular mass index was lower in patients under corticosteroid therapy but it was not low in previous corticosteroid users. Midtbo et al. [24] compared active RA patients and patients in remission and found out that higher disease activity is associated with greater left ventricular wall 
thickness. In the light of this result it is logical to think that left ventricular mass might be increased due to inflammation-induced vascular stiffening in RA patients and current medication might affect left ventricular mass index [22-24]. In our study, the majority of RA patients were under corticosteroid treatment. But we found out increased left ventricular mass index results which contradicts the results of Myasodeva et al. Further studies should be done to clarify the effect of corticosteroids on left ventricular mass index. In our study the disease and control groups were similar in terms of hypertension, body-mass index or other demographic characteristics so these basic characteristics should not cause the left ventricular mass index difference between the groups. C-reactive protein, white blood cell and neutrophil counts were increased in RA patients as a result of increased activity of inflammation which might be the cause of the increased left ventricular mass index.

\section{Conclusions}

In conclusion we showed that echocardiographic diastolic dysfunction parameters such as E/A, E wave, e' values were lower and IVS, PW diameters were higher in RA patients. Also left ventricular mass index was increased in RA patients compared to normal population. Main limitation of this study was small sample size. Further studies with larger sample size should be planned to confirm the results of our study.

\section{References}

[1]Turesson, C., et al., "Extra-articular disease manifestations in rheumatoid arthritis: Incidence trends and risk factors over 46 years", Ann Rheum Dis, 62, 722-727, 2003.

[2]Wolfe, F., et al., "The mortality of rheumatoid arthritis", Arthritis Rheum, 37, 481-494, 1994.

[3]Solomon, D.H., et al., "Cardiovascular morbidity and mortality in women diagnosed with rheumatoid arthritis", Circulation, 107, 1303-1307, 2003.

[4]Kaplan, M.J., "Cardiovascular disease in rheumatoid arthritis", Curr Opin Rheumatol, 18, 289-297, 2006.

[5]Nicola, P.J., et al., "The risk of congestive heart failure in rheumatoid arthritis: a population based study over 46 years", Arthritis \& Rheumatology, 52, 412-420, 2005.

[6] Nicola, P.J., et al., "Contribution of congestive heart failure and ischemic heart disease to excess mortality in rheumatoid arthritis", Arthritis \& Rheumatism, 54, 60-67, 2006.

[7] Avina-Zubieta, J.A., "Risk of cardiovascular mortality in patients with rheumatoid arthritis: a meta-analysis of observational studies", Arthritis Rheum, 59, 1690-1697, 2008.

[8]Liao, K.P., Solomon, D.H., "Traditional cardiovascular risk factors, inflammation and cardiovascular risk in rheumatoid arthritis", Rheumatology, 52, 45-52, 2013.

[9]Daviz, J.M., et al., "A signature of aberrant immune responsiveness identifies myocardial dysfunction in rheumatoid arthritis", Arthritis Rheum, 63, 1497-1506, 2011.

[10]Tsang, T.S., "Left atrial volume as a morpho-physiologic expression of left ventricular diastolic dysfunction and relation to cardiovascular risk burden", Am J Cardiol, 90, 1284-1289, 2002.

[11]Little, W.C., Cheng, C.P., "Diastolic dysfunction”, Cardiol Rev, 6, 231-239, 1998.

[12]Devereux, R.B., et al., "Echocardiographic assessment of left ventricular hypertrophy: comparison to necropsy findings", Am J Cardiol, 57, 450-458, 1986.

[13]Yazmalar, L., et al., "Seasonal disease activity and serum vitamin D levels in rheumatoid arthritis, ankylosing spondylitis and osteoarthritis", Afr Health Sc, 13, 47-55, 2013. 
[14]Pincus, T., et al. "Taking mortality in rheumatoid arthritis seriously - predictive markers, socioeconomic status and comorbidity", J Rheumatol, 13, 841-845, 1986.

[15]Mutru, O., et al., "Cardiovascular mortality in patients with rheumatoid arthritis", Cardiology, 76, 71-77, 1989.

[16]Lebowits, W.B., "The heart in rheumatoid arthritis (rheumatoid disease). A clinical and pathological study of 62 cases", Ann Intern Med, 58, 102-123, 1963.

[17]Redfield, M.M., et al., "Burden of systolic and diastolic ventricular dysfunction in the community: appreciating the scope of the heart failure epidemic", JAMA, 289, 194-202, 2003.

[18]Bursi, F., et al., "Systolic and diastolic heart failure in the community", JAMA, 296, 2209-2216, 2006.

[19]Di Franco, M., et al., "Diastolic function abnormalities in rheumatoid arthritis. Evaluation By echo Doppler transmitral flow and pulmonary venous flow: relation with duration of disease", Ann Rheum Dis, 59, 227-229, 2000.

[20]Erdem, F., et al. "Epicardial fat thickness in patients with rheumatoid arthritis", Afr Health Sci, 15, 489-495, 2015.

[21]Udayakumar, N., et al., "Diastolic function abnormalities in rheumatoid arthritis: relation with duration of disease", Singapore Med J, 48, 537-542, 2007.

[22]Rudominer, R.L., et al., "Independent association of rheumatoid arthritis with increased left ventricular mass but not with reduced ejection fraction", Arthritis Rheum, 60, 22-29, 2009.

[23]Myasoedova, E., et al., "Brief report: rheumatoid arthritis is associated with left ventricular concentric remodeling: results of a populationbased cross-sectional study", Arthritis Rheum, 65, 17131718, 2013.

[24] Midtbø, H., et al., "Disease activity and left ventricular structure in patients with rheumatoid arthritis", Rheumatology (Oxford), 54, 511-519, 2015. 